\title{
Design of Well Control System Based on Single Chip Microcomputer
}

\author{
Zhang Bin \\ No. 3 Drilling Company, Daqing Oilfield Drilling \\ Engineering Company \\ Daqing, China, \\ 93625945@qq.com
}

\author{
Wu Mengmeng* \\ Electrical and Information Engineering Institute, \\ Northeast Petroleum University \\ Daqing, China \\ 5605384@qq.com \\ * Corresponding Author
}

\begin{abstract}
In the process of measuring the well depth and temperature, due to the uncertainty of the air density in the well, and the different reflection ability of different impedance of the material on the sound wave, the metrical error is usually large. In this paper, we designed the temperature control system based on STC89C52 Single Chip Microcomputer (SCM) and DS18B20 chip, which used the DS18B20 temperature sensor to measure the well temperature and transmitted the temperature to the single chip microcomputer. Furthermore, we used the ultrasound to simulate infrasound to measure the well depth, realizing the temperature data acquisition, reading, processing, LCD1602 display, and the design of the alarm system for well depth, enhancing the precision of parameter measurement, ensuring the stable operation of well control system.
\end{abstract}

Keywords-Single Chip Microcomputer; Well Control System; Automation; Acquisition; Measurement

\section{INTRODUCTION}

In the process of oil field extraction, oil reservoir engineers often need to understand the accurate temperature curve of each point of the producing well, and then accurately analyze the oil reservoir and forecast the trend of production with various mining methods. On the other hand, oil production engineer need to understand the temperature of production well and injection well to ensure mining in optimal reservoir dynamic circumstances [1,2,3]. Therefore, in the process of production, we need special instruments to measure the change of the well depth and temperature. Unfortunately, conventional test methods has flaws, such as high cost, large error and low precision, etc.

In recent years, with the continuous development of electronic technology, information technology is shocking and changing people's traditional ways of thinking, working, as well as all fields of today's society. Among them, the ultrasonic detection technology is one of using the ultrasonic propagation characteristic in medium (such as sound velocity, attenuation, reflection, acoustic impedance, etc.) to implement the measurement of the non-acoustic volume (such as density, concentration, strength, elasticity, hardness, viscosity, temperature, flow velocity, flow, liquid level, thickness, defects, etc.) $[4,5,6]$. Ultrasonic wave meets different interface during the propagation in the medium, and the phenomenon such as reflection, refraction, diffraction and attenuation will emerge, so that the amplitude, waveform, frequency, etc. of the propagation will have changed correspondingly $[7,8]$. Determine the change of the rule, we can know certain properties and internal structure of materials. At present, the ultrasonic ranging has been widely used in various occasions, such as car reversing, ocean survey, object recognition, industrial automatic control, construction engineering measurement and robot vision recognition, etc. $[9,10,11]$

This paper takes STC89C52 SCM as the center control unit, and implements that there will form echo signal when ultrasonic launching and encountering obstacles occur reflection, and according to the propagation velocity of ultrasonic in the medium and the time difference from launching to receiving the echo, calculate the distance from the start to the obstacles, to accomplish the design object.

The designed well control system mainly includes SCM control module, DS18B20 temperature measuring module, LCD display module, etc. System working principle is: using the DS18B20 single bus digital temperature sensor to measure the internal well temperature and transmit it to the single chip microcomputer. Using the ultrasound as the simulate infrasound to measure the well depth. This design uses the AT89C51 SCM to drive the digital temperature sensor $\mathrm{DS} 18 \mathrm{~B} 20$ to acquire, read, process the temperature data, and display it by LCD1602.

\section{THE OVERALL FRAMEWORK OF THE SYSTEM}

The hardware part is composed of ultrasonic transmitting and echo signal receiving, temperature measurement, display and alarm, power supply, etc. The system principle block diagram is shown in Fig. 1 .

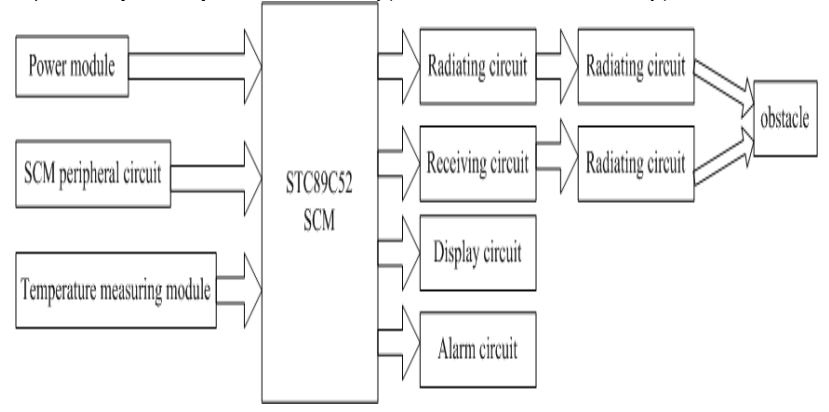

Figure 1. The Overall Framework Diagram of the Well Control System 
Among them, we select the STC89C52, and select DS18B20 as the temperature measurement module, whose principle diagram is shown in Fig. 2.

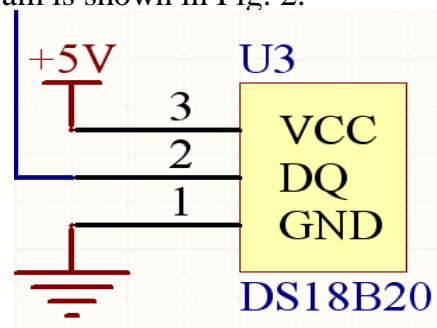

Figure 2. DS18B20 Temperature Measurement Principle Diagram

In LCD1602, we designed eight two-way communication mode. The wiring diagram is shown in Fig. 3.

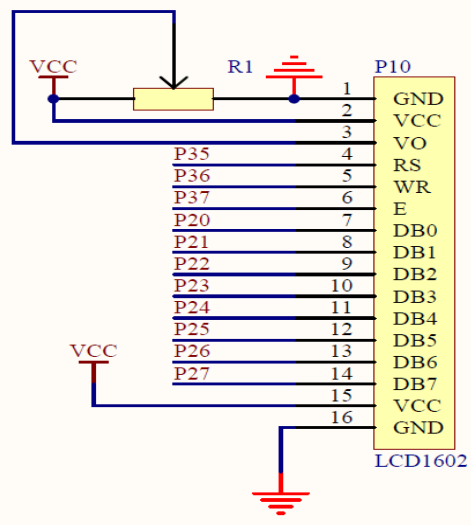

Figure 3. 1602 Wiring Diagram

The designed buzzer is active, whose connection way is shown in Fig. 4.

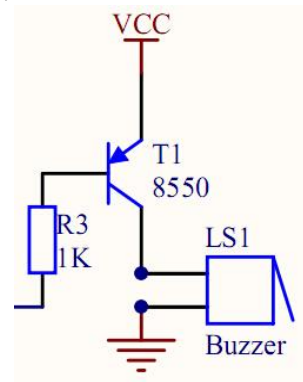

Figure 4. Buzzer Wiring Diagram

Ultrasonic ranging module is HC-SR04, and its advantages include stable performance, precise measure distance, and relatively small blind area, etc. If we make a console to send a high level of more than ten us, we can receive a high level output. When there is an output, we can open the timer. As the console turns to a low power level, we can read the value of the timer, and it is the time of this distance measurement, thus, we can calculate the distance. If we make circular measurement, we can achieve the value of the measurement.

\section{WELL CONTROL SYSTEM SCHEMATIC DIAGRAM}

The overall schematic diagram of the well control system is shown in Fig. 5.

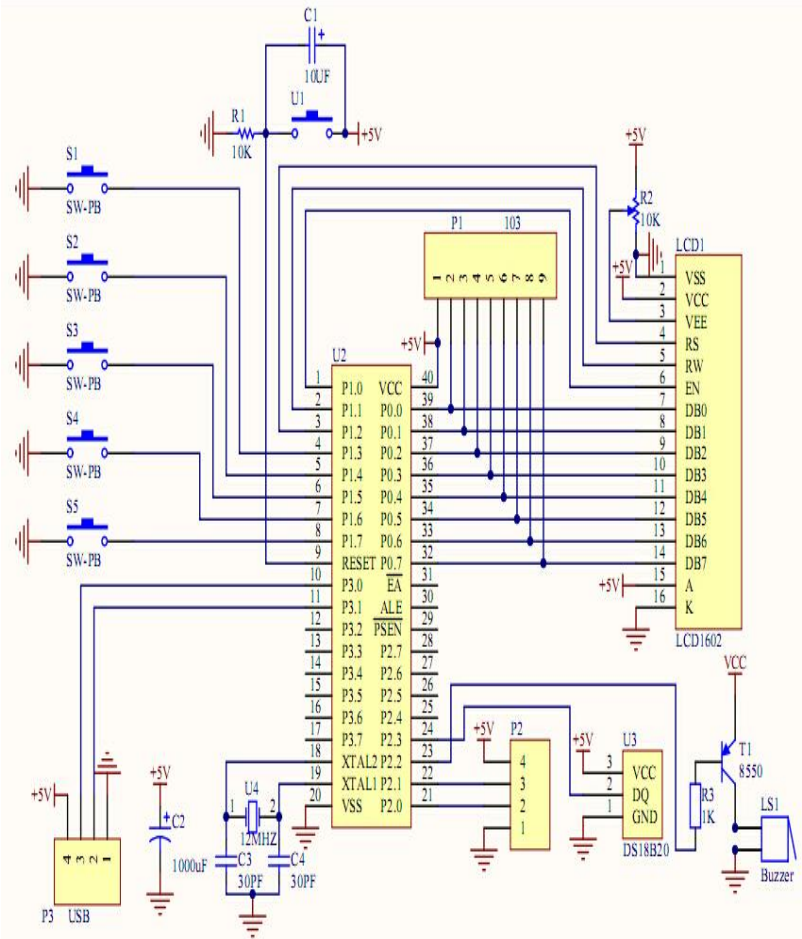

Figure 5. The Overall Schematic Diagram of the Well Control System

\section{SOFTWARE PROGRAM FLOW CHART DESIGN}

Software program is divided into the main program and subroutine, the main program invokes relevant subroutine to complete the overall program design, the main program flow chart is shown in Fig. 6.

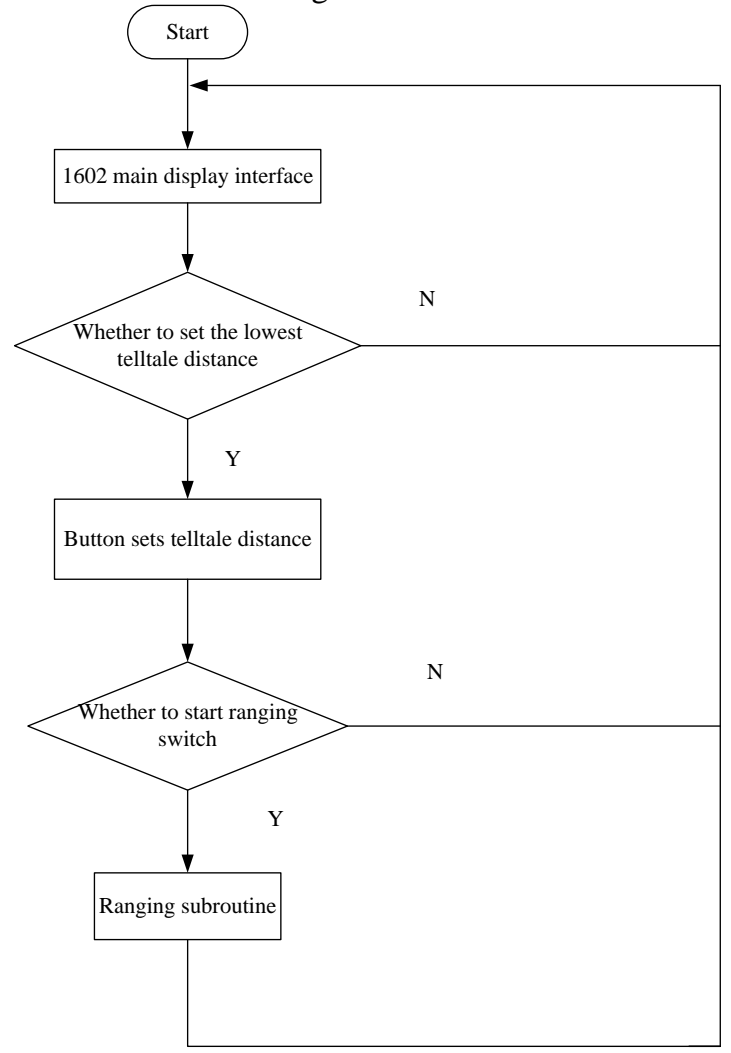

Figure 6. The Main Program Flow Chart 
Distance measurement subroutine is used to measure the well depth. When the main program runs and enters into the subroutine, the firstly running is to judge whether to press the self-locking switch which is used to start the ultrasonic ranging module to measure the distance. After the switch is pressed, we begin to measure the distance. We should judge whether the metrical distance value is less than the specific shortest threshold distance, if it is shorter than the specific distance, the buzzer will turn on the alarm, if it is greater than the specific temperature, the buzzer will not turn on the alarm. The corresponding distance measurement subroutine flow block diagram is shown in Fig. 7.

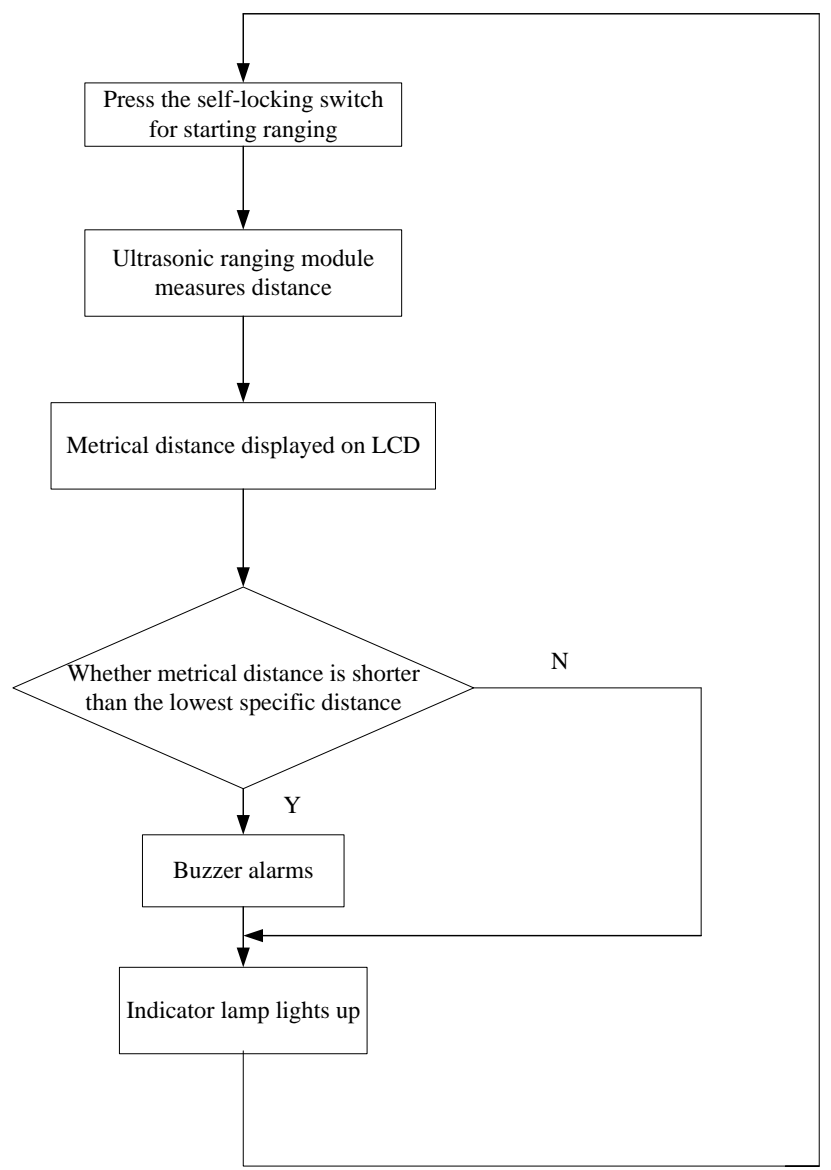

Figure 7. Distance Measurement Subroutine Flow Block Diagram

Display subroutine includes the writing console, writing data, reading status, etc. Before operating the LCE1602, we need to read the status. If the status is busy, we have to wait for a certain length of time, otherwise, we can write the data or write the console. The corresponding display subroutine flow block diagram is shown in Fig. 8.

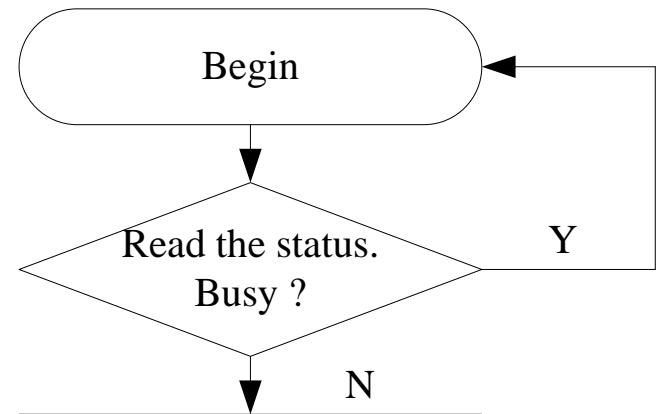

Set the way of displaying the LCD 1602

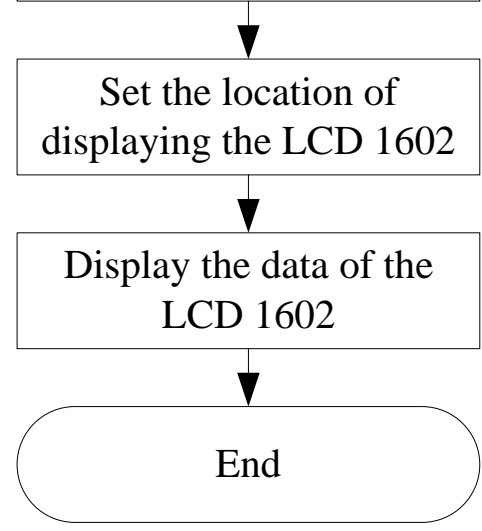

Figure 8. Display Subroutine Flow Block Diagram

\section{PICTURE OF REAL PRODUCTS AND THE ANALYSIS OF SYSTEM PERFORMANCE}

The picture of real products is shown in Fig. 9.

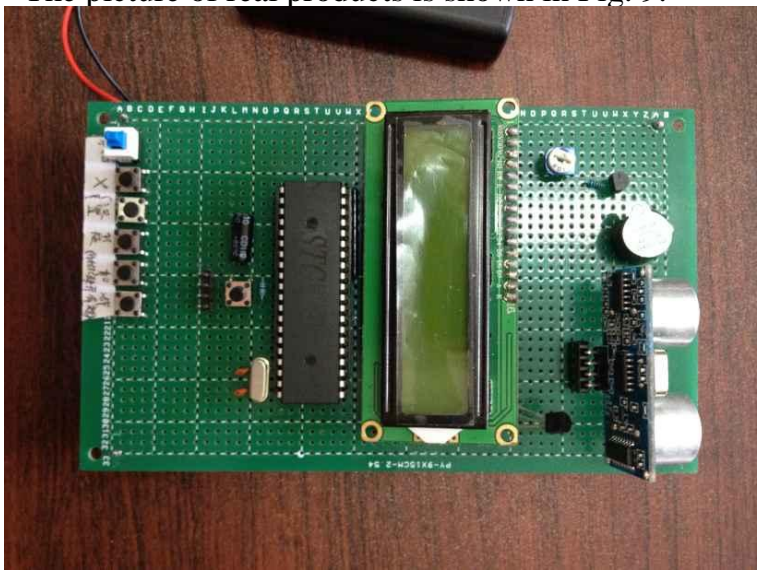

Figure 9. Picture of Real Products

The use of ultrasonic sensors HC-SRO4 and the temperature sensor DS18B20 offers many advantages to the assembly of the material object, which enables the designed system works stably for the environment in internal well.

The system is based on STC89C52 SCM, whose antiinterference ability enhances the operation reliability of the system effectively, while ultrasonic sensor HCSR04 and temperature sensor DS18B20 provide a possibility for data remote monitoring. Furthermore, the measured distance and the temperature are transmitted from the sensor to 
SCM, which, after sorted by SCM, are displayed on the LCD1602. In this way, we can know all the data of the internal well indoors.

\section{CONCLUSIONS}

The designed well control system based on SCM is one that is realized by measuring the distance and the temperature using ultrasonic ranging module and temperature sensor, and processing by SCM, displaying on the monitor. If the measured value is greater than the specific distance, the alarm will be turned on, if it isn't greater than the specific distance, it will normally work. In this paper, we designed the control system, which includes SCM and the design of the link of the hardware parts and the main circuit diagram, realizing the design of the software flow chart. Finally, through the simulation to check the effect of the control scheme and the feasibility of the material object. The system has certain practical value for improving the measuring accuracy of well depth and well temperature.

\section{ACKNOWLEDGMENT}

This work is partially supported by the Heilongjiang Provincial Natural Science Foundation for Young Scholars under Grant No. QC2014C066.

\section{REFERENCES}

[1] Li, Jian, "Design and development of single chip microcomputer (SCM) general experimental platform", Applied Mechanics and Materials, Vol. 195-196, pp. 885-888, 2012.

[2] Wang, Kun, Liu, Yunhong, "Remote experimental and development system of single chip microcomputer", In Proceedings of 2012 7th International Conference on Computer Science and Education, pp. 581-584, 2012.
[3] Su, Bing Ling, "Research and development of agricultural machinery operating area measuring system based on single chip computer", Advanced Materials Research, Vol. 912-914, pp. $994-$ 997, 2014.

[4] Lahiri, C., Knobloch, K., Bake, F., Enghardt, L., "Attenuation of sound in wide ducts with flow at elevated pressure and temperature", Journal of Sound and Vibration, No. 15, pp. 34403458, 2014

[5] Radosz, Jan, "Uncertainty due to instrumentation for sound pressure level measurement in high frequency range", Noise Control Engineering Journal, Vol. 62, pp. 186-195, 2014.

[6] Annibale, Paolo, Filos, Jason, Naylor, Patrick A., Rabenstein, Rudolf, "TDOA-based speed of sound estimation for air temperature and room geometry inference", IEEE Transactions on Audio, Speech and Language Processing, Vol. 21, pp. 234-246, 2013.

[7] Fu, Qiang, Li, Min, Wei, Long, Yang, Debin. "An improved method combining beamforming and acoustical holography for the reconstruction of the sound pressure on structure surface", Acta Acustica united with Acustica, Vol. 100, pp. 166-183, 2014.

[8] Hui Wu, Jiu, Hu, Zhi Ping, Zhou, Han, "Sound absorbing property of porous metal materials with high temperature and high sound pressure by turbulence analogy", Journal of Applied Physics, Vol. $113,2013$.

[9] Elshenawy, Tamer, Li, Q.M., "Influences of target strength and confinement on the penetration depth of an oil well perforator", International Journal of Impact Engineering, Vol. 54, pp. 130-137, 2013.

[10] Li, Hongtao, Meng, Yingfeng, Li, Gao, Wei, Na, Liu, Jiajie, Ma Xiao, Duan, Mubai, Gu, Siman, Zhu, Kuanliang, Xu, Xiaofeng, "Propagation of measurement-while-drilling mud pulse during high temperature deep well drilling operations", Mathematical Problems in Engineering, Vol. 2013, 2013.

[11] Durocher, Stephane, Fraser, Robert, Leblanc, Alexandre, Morrison Jason, Skala, Matthew, "On combinatorial depth measures", 26th Canadian Conference on Computational Geometry, CCCG 2014 pp. 198-205, 2014. 